PROCEEDINGS OF THE

AMERICAN MATHEMATICAL SOCIETY

Volume 130, Number 12, Pages 3579-3583

S 0002-9939(02)06667-4

Article electronically published on June 18, 2002

\title{
RELAXATION LIMIT FOR HYPERBOLIC SYSTEMS IN CHROMATOGRAPHY
}

\author{
YUN-GUANG LU
}

(Communicated by David S. Tartakoff)

\begin{abstract}
This paper is concerned with a $2 n \times 2 n$ nonlinear system which arises in chromatography. The global existence of solutions $\left(u_{i}^{\tau}, v_{i}^{\tau}\right)$ in $L^{\infty}$ space for a Cauchy problem with initial data is obtained for any fixed $\tau>$ 0 , and the convergence of $\left(u_{i}^{\tau}, v_{i}^{\tau}\right)$ to its equilibrium state $\left(u_{i}, v_{i}\right)$, governed by a limit system is proved for the case $n=2$ by using the compensated compactness coupled with the framework of Tzavaras (1999).
\end{abstract}

\section{INTRODUCTION}

We are concerned with the Cauchy problem for $2 n \times 2 n$ nonlinear system

$$
\left\{\begin{array}{l}
u_{i t}+u_{i x}+\frac{F_{i}(u)-v_{i}}{\tau}=0, \\
v_{i t}+\frac{v_{i}-F_{i}(u)}{\tau}=0,
\end{array}\right.
$$

with initial data

$$
\left(u_{i}(x, 0), v_{i}(x, 0)\right)=\left(u_{i 0}(x), v_{i 0}(x)\right)
$$

where $i=1,2, \ldots, n$ and $\tau>0$. (1.1) arises in chromatography 9 , where $u_{i}$ represents the concentration of the solute in the fluid phase and $v_{i}$ its concentration in the solid phase, both being expressed in moles per unit volume of their own phase. When $\tau=0$, the equilibrium relation $v_{i}$ is usually called the adsorption isotherm, and in general, it is a complicated nonlinear function of $u_{i}$ in which mutual influences among different solutes are taken into account. In this paper, our interest is to restrict on the special equilibrium relation

$$
v_{i}=F_{i}(u)=u_{i} \phi(r), \quad r=\sum_{i=1}^{n} u_{i}^{2}
$$

We study the existence of global solutions $\left(u_{i}^{\tau}, v_{i}^{\tau}\right)$ for Cauchy problem (1.1), (1.2) and their convergence, as $\tau$ tends to zero, to its equilibrium state $\left(u_{i}, v_{i}\right)$, governed by the limit system

$$
\left(u_{i}+u_{i} \phi(r)\right)_{t}+u_{i x}=0, \quad i=1,2, \ldots, n .
$$

System (1.4) itself is of interest since it arises in such areas as elasticity theory, magnetohydrodynamics, and enhanced oil recovery ([1, 2, 4, 9, 11]). When $n=1$,

Received by the editors March 24, 2001.

2000 Mathematics Subject Classification. Primary 35L65, 35B40.

Key words and phrases. Multicomponent chromatography, relaxation limit, compensated compactness. 
the convergence and the error estimates of solutions $\left(u^{\tau}, v^{\tau}\right)$ to the unique weak solution $(u, v)$ of the scalar equation

$$
(u+F(u))_{t}+u_{x}=0
$$

have been well studied by many authors (see [7, 11]). However the case of $n \geq 2$ is open except for some numerical results 9] and the recent works on $B V$ solutions for small initial data [1].

In this paper we have the following main results.

Theorem 1. Let $\phi \in C^{1}, \phi^{\prime}(r)>0$ for $r \in[0, \infty)$ and its inverse function be $\phi^{-1}(r) ; \phi(r) \rightarrow \infty$ as $r \rightarrow \infty$. If the initial data $\left(u_{i 0}(x), v_{i 0}(x)\right)$ are bounded, $\phi\left(r_{0}(x)\right) \leq d, s_{0}(x) \leq d^{2} \phi^{-1}(d)$ for a positive constant $d$, where $s=\sum_{i=1}^{n} v_{i}^{2}$, then for any fixed $\tau>0$, Cauchy problem (1.1), (1.2) has a unique, global classical solution $\left(u_{i}^{\tau}, v_{i}^{\tau}\right)$, on $R \times[0, T]$, for any given $T>0$, which satisfies

$$
\left|u_{i}^{\tau}\right|+\left|v_{i}^{\tau}\right| \leq M
$$

where $M$ is a positive constant which depends only on the initial data.

Theorem 2. Let the initial data $\left(u_{i 0}(x), v_{i 0}(x)\right)$ have compact support or tend to zero sufficiently fast as $|x| \rightarrow \infty$. If $n=2$ and $\phi(r) \geq c$ for a positive constant $c$, then there exists a subsequence, still denoted by $\left(u_{i}^{\tau}, v_{i}^{\tau}\right)$, of the solutions of (1.1), (1.2), which converges to bounded functions $\left(u_{i}, v_{i}\right)$ as $\tau \rightarrow 0$, where $v_{i}=u_{i} \phi(r)$, a.e. and $u_{i}$ is a weak solution of equilibrium system (1.4).

The main ideas for the proofs of Theorems 1 and 2 come from the recent investigations of $\mathrm{A}$. Tzavaras on a $3 \times 3$ system in $L^{p}$ space 12 . The convergence of the relaxation solutions to its equilibrium state is based on the compensated compactness. This method was well used in the relaxation problems for hyperbolic conservation laws of two equations in combustion theory, multiphase and phase transition [4, viscoelasticity [3], and of three equations in chemical reaction flows 12].

\section{Proofs of Theorems 1 And 2}

In this section, we give the proofs of Theorems 1 and 2 stated in Section 1.

Proof of Theorem 1. Theorem 1 can be proved via the existence of a local solution of $(1.1),(1.2)$ (see [10, 12]) and the a-priori $L^{\infty}$ estimates given in (1.6).

To prove (1.6), we multiply the first and second equations in (1.1) by $2 u_{i}, 2 v_{i}$ respectively, and obtain

$$
\left\{\begin{array}{l}
r_{t}+r_{x}+\frac{2 r \phi(r)-\sum_{i=1}^{n} 2 u_{i} v_{i}}{\tau}=0 \\
s_{t}+\frac{2 s-\sum_{i=1}^{n} 2 u_{i} v_{i} \phi(r)}{\tau}=0 .
\end{array}\right.
$$

From (2.1), we immediately obtain the following inequalities:

$$
\left\{\begin{array}{l}
r_{t}+r_{x}+\frac{r(2 \phi(r)-d)-s / d}{\tau} \leq 0 \\
s_{t}+\frac{s-r(\phi(r))^{2}}{\tau} \leq 0
\end{array}\right.
$$


for a constant $d$ since

$$
\sum_{i=1}^{n} 2 u_{i} v_{i} \leq d r+\frac{s}{d}, \quad \sum_{i=1}^{n} 2 u_{i} v_{i} \phi(r) \leq s+r(\phi(r))^{2} .
$$

Let $(\bar{r}, \bar{s})$ satisfy

$$
r(2 \phi(r)-d)-s / d=0, \quad s-r(\phi(r))^{2}=0 .
$$

Then by simple calculations,

$$
\phi(\bar{r})=d, \quad \bar{r}=\phi^{-1}(d), \quad \bar{s}=d^{2} \phi^{-1}(d) .
$$

Noticing the conditions on the initial data, we can see that $r=\bar{r}, s=\bar{s}$ is a supersolution of (2.1). Thus we have

$$
r \leq \bar{r}, \quad s \leq \bar{s}
$$

which imply (1.6) and the proof of Theorem 1.

Proof of Theorem 2. The proof of Theorem 2 is based on the following estimates on the relaxation approximated solutions $\left(u_{i}^{\tau}, v_{i}^{\tau}\right)$ of Cauchy problem $(1.1),(1.2)$ :

$$
\begin{gathered}
\tau\left|u_{i t}^{2}\right|_{L^{1}(R \times[0, T])} \leq M, \\
\tau\left|u_{i x}^{2}\right|_{L^{1}(R \times[0, T])} \leq M, \\
\left|u_{i} \phi(r)-v_{i}\right|_{L^{2}(R \times[0, T])} \leq \tau M, \\
\tau\left|v_{i t}^{2}\right|_{L^{1}(R \times[0, T])} \leq M .
\end{gathered}
$$

To prove (2.7), we add two equations in (1.1) together and then use the first equation again, and obtain

$$
\left(u_{i}+u_{i} \phi(r)\right)_{t}+u_{i x}+\tau\left(u_{i t}+u_{i x}\right)_{t}=0 .
$$

Multiplying (2.11) by $2 u_{i}$ and $2 \tau u_{i t}$ respectively, we have

$$
\left(r+2 r \phi(r)-\int_{0}^{r} \phi(r) d r\right)_{t}+r_{x}+\tau\left(r_{t}+r_{x}\right)_{t}-2 \tau \sum_{i=1}^{n}\left(u_{i t}+u_{i x}\right) u_{i t}=0
$$

and

$$
2 \tau \sum_{i=1}^{n}\left(u_{i t}+u_{i x}\right) u_{i t}+2 \tau \sum_{i=1}^{n} u_{i t}\left(u_{i} \phi(r)\right)_{t}+\tau^{2} \sum_{i=1}^{n}\left(\left(u_{i t}^{2}\right)_{t}+\left(u_{i t}^{2}\right)_{x}\right)=0 .
$$

Adding up (2.12) and (2.13) gives

$$
\begin{aligned}
& \left(r+\tau r_{t}+\tau^{2} \sum_{i=1}^{n} u_{i t}^{2}\right)_{t}+\left(2 r \phi(r)-\int_{0}^{r} \phi(r) d r\right)_{t} \\
& \quad+\left(r+\tau r_{t}+\tau^{2} \sum_{i=1}^{n} u_{i t}^{2}\right)_{x}+2 \tau \sum_{i=1}^{n} u_{i t}\left(u_{i} \phi(r)\right)_{t}=0 .
\end{aligned}
$$

Since

$$
\begin{gathered}
r+\tau r_{t}+\tau^{2} \sum_{i=1}^{n} u_{i t}^{2}=\sum_{i=1}^{n}\left(u_{i}^{2}+2 \tau u_{i} u_{i t}+\tau^{2} u_{i t}^{2}\right) \geq 0, \\
2 r \phi(r)-\int_{0}^{r} \phi(r) d r \geq 0,
\end{gathered}
$$


and

$$
\sum_{i=1}^{n} u_{i t}\left(u_{i} \phi(r)\right)_{t}=\sum_{i=1}^{n} u_{i t}^{2} \phi(r)+2 \phi^{\prime}(r)\left(\sum_{i=1}^{n} u_{i} u_{i t}\right)^{2} \geq c \sum_{i=1}^{n} u_{i t}^{2},
$$

we integrate (2.14) on $R \times[0, T]$ and then get the proof of (2.7). To prove (2.8), we multiply (2.11) by $\tau u_{i x}$ and obtain

$$
\begin{gathered}
\tau \sum_{i=1}^{n} u_{i x}^{2}+\tau \sum_{i=1}^{n} u_{i x}\left(u_{i}+u_{i} \phi(r)\right)_{t}+\tau^{2} \sum_{i=1}^{n}\left(u_{i x} u_{i t}\right)_{t} \\
-\frac{1}{2} \tau^{2} \sum_{i=1}^{n}\left(\left(u_{i t}^{2}\right)_{x}-\left(u_{i x}^{2}\right)_{t}\right)=0
\end{gathered}
$$

Integrating (2.18) on $R \times[0, T]$ and noticing the boundedness of $\tau u_{i t}^{2}$ in $L^{1}(R \times[0, T])$, we obtain

$$
\int_{-\infty}^{\infty} \tau^{2} \sum_{i=1}^{n}\left(\frac{1}{2} u_{i x}^{2}+u_{i x} u_{i t}\right) d x+\int_{0}^{T} \int_{-\infty}^{\infty} \frac{\tau}{2} \sum_{i=1}^{n} u_{i x}^{2} d x d t \leq M .
$$

From (2.13), we have

$$
\int_{-\infty}^{\infty} \frac{1}{2} \tau^{2} \sum_{i=1}^{n} u_{i t}^{2} d x \leq M_{1}+\int_{0}^{T} \int_{-\infty}^{\infty} \frac{\tau}{4} \sum_{i=1}^{n} u_{i x}^{2} d x d t .
$$

Adding up (2.19) and (2.20), we have

$$
\int_{-\infty}^{\infty} \frac{1}{2} \tau^{2} \sum_{i=1}^{n}\left(u_{i t}+u_{i x}\right)^{2} d x+\frac{\tau}{4} \int_{0}^{T} \int_{-\infty}^{\infty} \sum_{i=1}^{n} u_{i x}^{2} d x d t \leq M_{2}
$$

which implies (2.8).

From $(2.7),(2.8)$ and the first equation in (1.1), we can prove (2.9). Using the second equation in (1.1) and estimates (2.9), we get (2.10).

From estimates $(2.7)-(2.10)$ and the standard method given in [3, 4], we can prove that

$$
\eta\left(u_{1}^{\tau}, u_{2}^{\tau}\right)_{x}+q\left(u_{1}^{\tau}, u_{2}^{\tau}\right)_{t} \quad \text { is compact in } \quad H_{l o c}^{-1}\left(R \times R^{+}\right),
$$

where $(\eta, q)$ is any entropy-entropy flux pair of (1.4) constructed in 2 .

Finally, using the convergence framework in [2], we get the compactness of

$$
\left(u_{1}^{\tau}, u_{2}^{\tau}\right) \rightarrow\left(u_{1}, u_{2}\right) \text { a.e. on } \Omega,
$$

where $\Omega$ is any bounded open subset of $R \times R^{+}$. Noticing estimates (2.9), we get the equilibrium relation

$$
v_{i}=u_{i} \phi(r), \quad \text { a.e. on } \Omega,
$$

where $i=1,2$. Therefore, Theorem 2 is proved.

\section{ACKNOWLEDGEMENT}

This paper was partially supported by a grant from the National University of Colombia, Bogota, and NNSF 10071080 from China. 


\section{REFERENCES}

[1] A. Bressan and W. Shen, Estimates for Multicomponent Chromatography with Relaxation, Discr. Cont. Dyn. Sys., 6(2000), 21-38. MR 2000m:35121

[2] G. Q. Chen, Hyperbolic system of conservation laws with asymmetry, Commun. PDE, 16(1991), 1461-1487. MR 92g:35130

[3] G. Q. Chen, C.D. Levermore and T. P. Liu, Hyperbolic conservation laws with stiff relaxation terms and entropy, Comm. Pure Appl. Math., 47(1994), 787-830. MR 95h:35133

[4] G. Q. Chen and T. P. Liu, Zero relaxation and dissipation limits for hyperbolic conservation laws, Comm. Pure Appl. Math., 46(1993), 755-781. MR 94b:35167

[5] H. A. Freistühler, Standard model of generic rotational degeneracy, In: Nonlinear Hyperbolic Equations, Theory, Computation Methods and Applications, edited by J. Ballmann and R. Jeltsch, Vieweg, Braunschweig 1989, pp. 149-158. MR 90k:35166

[6] B. Keyfitz and H. Kranzer, A system of non strictly hyperbolic conservation laws arising inelasticity, Arch. Rational Mech. Anal., 72(1980), 219-241. MR 80k:35050

[7] A. Kurganov and E. Tadmor, Stiff system of hyperbolic conservation laws: Convergence and error estimates, SIAM J. Math. Anal., 28(1997), 6: 1446-1456. MR 98j:35117

[8] T. P. Liu and J. H. Wang, On a hyperbolic system of conservation laws which is not strictly hyperbolic, J. Diff. Equs., 57(1985), 1-14. MR 86j:35108

[9] H. K. Rhee, R. Aris and N. R. Amundsen, On the theory of multicomponent chromatography, Phil. Trans. Royal. Soc. of London, 267A (1970), 419-455.

[10] J. A. Smoller, Shock Waves and Reaction-Diffusion Equations, Springer Verlag, New YorkHeidelberg-Berlin (1982). MR 84d:35002

[11] A. Tveito and R. Winther, On the rate of convergence to equilibrium for a system of conservation laws including a relaxation term, SIAM J. Math. Anal., 28(1997), 136-161. MR 98a:35087

[12] A. Tzavaras, Materials with internal variables and relaxation to conservation laws, Arch. Rational Mech. Anal., 146(1999), 2, 129-155. MR 2000i:74004

Departamento de Matemáticas y Estadística, Universidad Nacional de Colombia, Bogotá, Colombia - and - Department of Mathematics, University of Science and Technology of China, Hefei, People's Republic of China

E-mail address: yglu@matematicas.unal.edu.co 